\title{
Quiste de vallécula como causa de estridor congénito. Reporte de un caso y revisión de la literatura
}

\section{Vallecula cyst as a cause of congenital stridor. Case report and literature review}

\author{
Jaime Osorio $\mathbf{M}^{1}$, Miguel Cancino $\mathbf{C}^{1}$, Rodrigo Arregui V1, Angélica Suazo D².
}

\begin{abstract}
RESUMEN
El quiste de vallécula congénito es una malformación de vía aérea poco frecuente que se manifiesta principalmente con estridor laríngeo y que puede plantear problemas diagnósticos y terapéuticos complejos, a menudo en situaciones de riesgo vital. Se presenta el caso de un recién nacido de pretérmino (RNPT) de 36 semanas pequeño edad gestacional (PEG), portador de un estridor laríngeo congénito y mal incremento pondoestatural que en relación a una infección respiratoria baja, presentó empeoramiento del estridor laríngeo a los 48 días de edad cronológica. Por insuficiencia respiratoria aguda requirió de intubación orotraqueal que no resultó dificultosa. La extubación fue fallida por presentar estridor inspiratorio franco. La nasofibroscopía demostró una lesión de aspecto quístico en base de lengua que desplazaba la epiglotis hacia posterior obstruyendo parcialmente el lumen de la vía aérea. Por laringoscopía directa se realizó marsupialización. Se realiza la revisión bibliográfica y se analiza el caso y su tratamiento.
\end{abstract}

Palabras clave: Quiste laríngeo congénito, quiste de vallécula, estridor congénito.

\begin{abstract}
Congenital vallecular cyst is a rare airway malformation mainly manifested by laryngeal stridor and could generate complex diagnostic and therapeutic problems, often in life-threatening situations. We present the case of a pre-term newborn of 36 weeks small for gestational age, who at 48 days of chronological age showed worsening of a congenital laryngeal stridor in the context of a lower respiratory tract infection associated to low weight gain from birth. For reasons of acute respiratory failure, orotracheal intubation was executed which was not difficult. Extubation was failed because the child presents significant inspiratory stridor. Nasofibroscopy showed a cystic lesion of the tongue base that pushed backward the epiglottis obstructing partially the airway lumen. Marsupialization was performed by direct laryngoscopy. A Bibliographic review was done and the case and its treatment are discuss
\end{abstract}

Key words: Congenital laryngeal cyst, vallecula cyst, congenital stridor.

1 Médico Servicio de Otorrinolaringología, Hospital Barros Luco Trudeau. Universidad de Chile.

2 Alumna de Medicina. Universidad Católica de la Santísima Concepción. 


\section{INTRODUCCIÓN}

Las malformaciones congénitas de la laringe (MCL) son infrecuentes, algunas de difícil diagnóstico y tratamiento. Su sintomatología es diversa, pero destaca el estridor, el distrés respiratorio, la disnea obstructiva, la disfonía y el trastorno de la deglución ${ }^{1-2}$. El diagnóstico se basa en la exploración de la vía aérea con nasofibroscopio y/0 en caso necesario, laringoscopía directa 0 broncoscopía rígida ${ }^{3}$.

Entre las MCL se incluyen los quistes laríngeos congénitos, con una incidencia de 2 por cada 300.000 recién nacidos vivos ${ }^{4}$. Fueron descritos por primera vez en 1881 por Abercrombie. De Santo y col, en 1970, clasificaron a los quistes laríngeos congénitos en saculares 0 del foramen del cartílago tiroides y ductales.

El quiste sacular corresponde a una distensión mucosa del sáculo laríngeo como consecuencia de una atresia u obstrucción del orificio ventricular; y cuando traspasa el inconstante foramen del cartílago tiroides, reciben el nombre de quiste del foramen del cartílago tiroides ${ }^{5}$. Los quistes ductales corresponden a una distensión mucosa de una glándula salival menor producto de la obstrucción de su ducto de drenaje, con moco blanquecino denso en su interior y representan el $75 \%$ de los casos. Su ubicación más habitual es la vallécula 0 la base de la lengua, por lo que habitualmente el término de quiste ductal se reserva al ámbito anatomopatológico ${ }^{6}$.

Aun cuando los quistes valleculares se encuentran ubicados fuera de la laringe son considerados en la literatura científica como quistes laríngeos congénitos debido a que pueden desplazar hacia posterior la epiglotis y obstruir la estrecha vía aérea del neonato $0^{6,7}$.

El objetivo del artículo es reportar un caso y revisar la literatura al respecto.

\section{PRESENTACIÓN DEL CASO}

Se presenta el caso de un lactante menor con antecedente de RNPT 36 semanas PEG, producto de un embarazo no controlado, con mal incremento ponderal, alimentándose solo con fórmula. Consulta a los 48 días de nacido en el Servicio de Urgencia del Hospital Pediátrico Exequiel González Cortés por un cuadro de 4 días de evolución caracterizado por estornudos, rinorrea acuosa y tos que progresivamente se hace húmeda y en accesos, en ocasiones emetizante, asociado a rubicundez $\mathrm{y} / 0$ cianosis facial. Siempre afebril. Dirigídamente se obtiene historia de sutil estridor con el llanto y durante la lactancia desde la segunda semana de vida. Se hospitaliza para manejo con oxígeno suplementario, broncodilatadores B2 agonistas y corticoides sistémicos. Evoluciona con aumento de la dificultad respiratoria llegando a requerir $\mathrm{FiO}_{2}$ de $40 \%$. Se decide traslado a Unidad de Paciente Crítico. En esta unidad evoluciona sin signología obstructiva baja, por lo que se suspenden los broncodilatadores y la terapia corticoesteroidal, progresando con estridor inspiratorio y apremio respiratorio evidente, por lo cual es conectado a C-PAP. A pesar de lo anterior, en 24 horas genera acidosis respiratoria, por lo que se decide el cambio a sistema B-PAP, con lo que disminuye claramente el estridor y la dificultad respiratoria, mejorando a su vez los niveles de $\mathrm{PCO}_{2}$ arteriales. Sin embargo tres días después por empeoramiento del estridor inspiratorio requirió ventilación mecánica invasiva por gran dificultad respiratoria. Se realiza fibrobroncoscopía por pediatra broncopulmonar donde se describe acceso dificultoso por vía nasal hacia laringe por eplíglotis elongada y en omega que oblitera glotis. Se instala máscara laríngea por la cual acceden a laringe y tráquea, describiendo leve traqueomalacia distal. Ante la necesidad de evaluar detalladamente la anatomía laríngea se interconsulta a otorrinolaringología, realizándose una nasofibroscopía extubada y sedada apreciándose masa de aspecto quístico en la base de la lengua que toma contacto con epíglotis en omega y la desplaza obstruyendo el lumen de la vía aérea. Se requiere la reintubación orotraqueal por desaturación crítica. Con diagnóstico de quiste preepiglótico se programa intervención quirúrgica, la cual se realiza por laringoscopía directa con óptica endoscópica de $0^{\circ}$. En base de lengua al llegar a vallécula se observa lesión quística redondeada en línea media que desplaza epíglotis hacia anterior (Figura 1). Se observan signos inflamatorios 


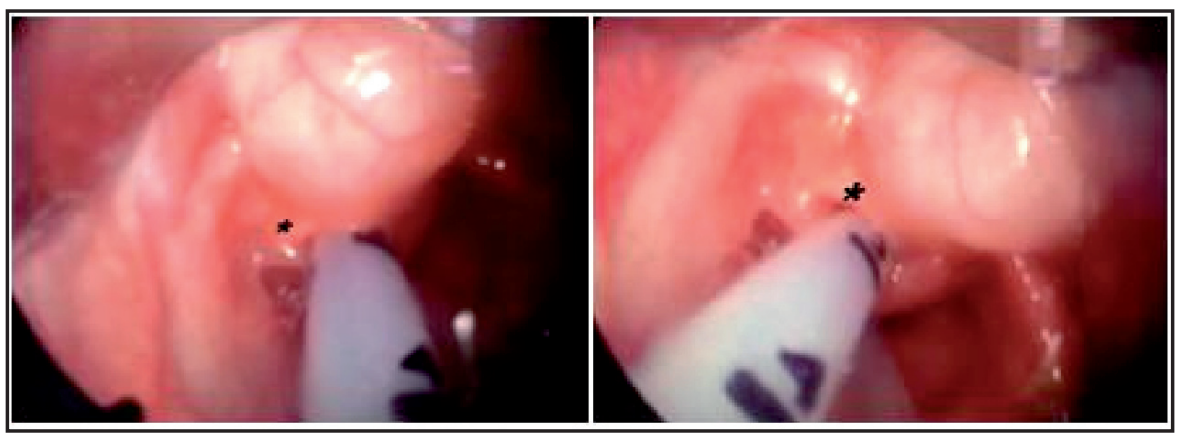

Figura 1. Quiste de vallécula que desplaza a la epíglotis $\left(^{*}\right)$ hacia posterior, obstruyendo la vía aérea.

en aritenoides y supraglotis. Cuerdas vocales de aspecto normal, sin otra lesión asociada. Se realiza amplia marsupialización con técnica fría y control de hemostasia con electrocoagulador monopolar (Figura 2). Se extuba a los 4 días poscirugía sin incidentes, sin estridor residual. En control al sexto día poscirugía se aprecia paciente tranquila, sin dificultad respiratoria. A la nasofibroscopía en el lecho quirúrgico se observa escasa fibrina, sin edema. Biopsia fue informada como quiste ductal laríngeo sin atipias. Seguimiento a los 9 meses poscirugía demuestra ausencia de recidiva a la nasofibrosocopía (Figura 3) y paciente clínicamente asintomática.

\section{DISCUSIÓN}

Los quistes valleculares aunque raros, deben ser considerados en el diagnóstico diferencial

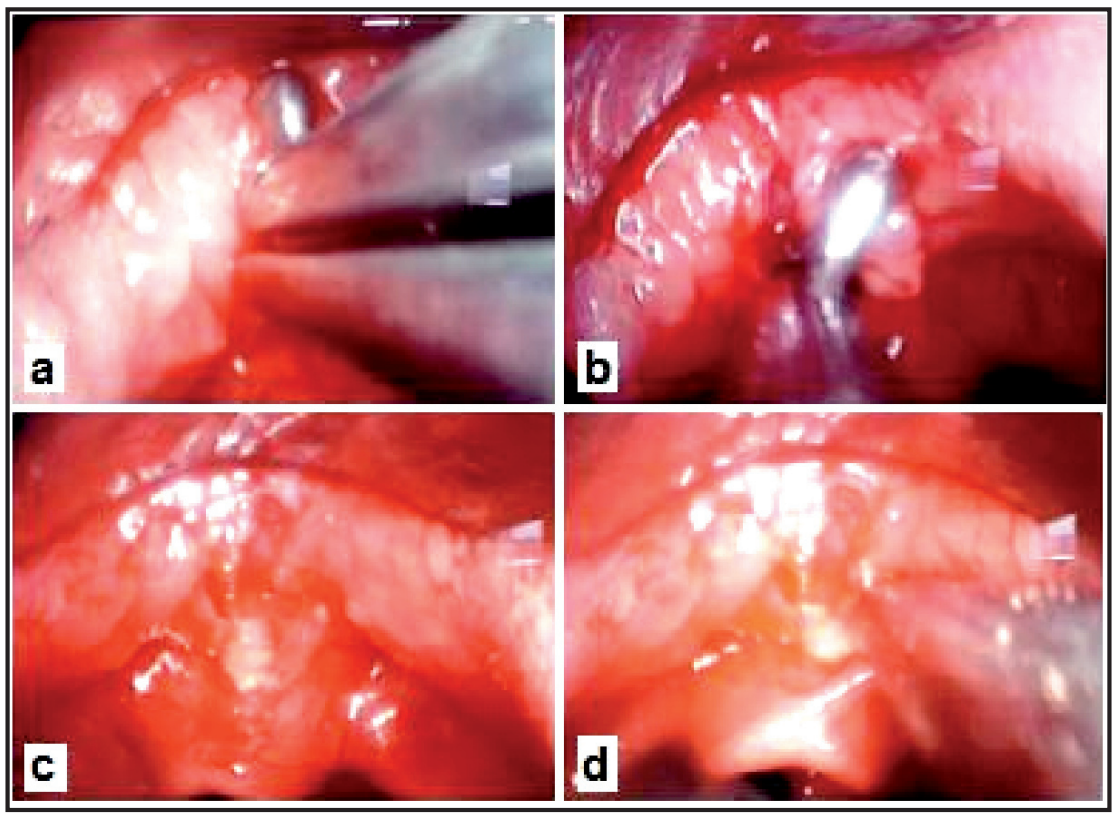

Figura 2. Abordaje del quiste de vallécula con técnica fría (a y b); realizando amplia marsupialización (c) y control de hemostasia con electrocauterio monopolar (d). 


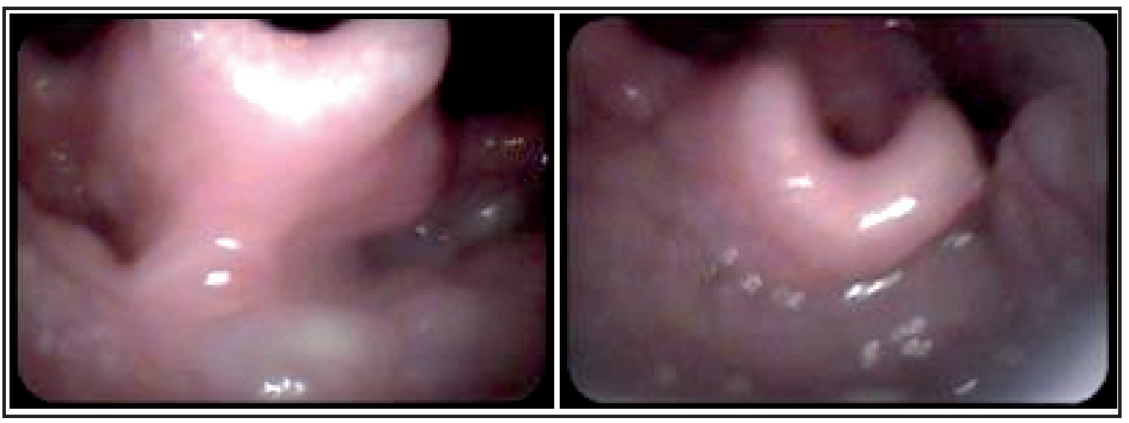

Figura 3. Imagen de control nasofibroscópico al noveno mes posquirúrgico. Se observa vallécula libre.

de un estridor neonatal y podrían ser la causa de una muerte súbita infantil ${ }^{7-8}$. En los adultos suelen ser asintomáticos o provocar síntomas inespecíficos, como disfonía, globo faríngeo 0 disfagia ${ }^{9}$. Constituyen la ubicación más frecuente de los quistes ductales laríngeos. Otros sitios son las cuerdas vocales, los repliegues ariepiglóticos y el seno piriforme $e^{1,6,7}$.

El diagnóstico puede ser hecho de forma prenatal desde la 25 semana de gestación con signos indirectos, como polihidroamnios, hipoplasia pulmonar o compresión traqueal7,10. Los síntomas dependerán de la localización y del tamaño del quiste vallecular; destacando estridor, distrés respiratorio, disnea, retracción intercostal, disfonía, crisis recurrentes de cianosis, tos crónica, mal incremento pondoestatural, disfonía, Ilanto anormal, disfagia y muerte súbita.

Estos pacientes pueden presentarse de forma asintomática al momento de nacer, pero con el paso de las semanas desarrollan sintomatología obstructiva que puede exacerbarse en el contexto de una infección del tracto respiratorio superior ${ }^{11-13}$.

Se relaciona con otras alteraciones de la vía aérea como laringomalacia en el $12 \%-45 \%$ y reflujo gastroesofágico en el $70 \%$ de los casos. La prematuridad no constituye factor de riesgo para presentarlo ${ }^{14-15}$.

Macroscópicamente, los quistes ductales valleculares son masas quísticas uniloculares de tamaño variable con contenido mucoso, denso, no infectado. Histológicamente, el quiste contiene epitelio respiratorio y glándulas mucosas, cubierto externamente por epitelio escamoso ${ }^{5}$.
La primera clasificación de los quistes laríngeos la realizó De Santo y cols en 1970 basado en su ubicación, distinguiéndolos así como ductales, saculares y quistes del foramen del cartílago tiroíde $0^{5}$. Posteriormente aparecen descripciones basadas en la histomorfología, como la de Newman $\mathrm{BH}$ y cols en 1984 y la de Ramesar K y Albizzati C en 1988; donde los clasifican en epiteliales, tonsilares y oncocíticos ${ }^{18-19}$. Fue Arens y cols en 1997 quien propone una nueva clasificación basada en la localización e histomorfología del quiste, clasificándolos como quistes congénitos, de retención y de inclusión ${ }^{20}$. Por último, Forte y cols en 2004 propusieron un nuevo sistema de clasificación para los quistes laríngeos congénitos con un enfoque terapéutico. Esta clasificación se basa en la extensión del quiste y en el origen embriológico del tejido. Así, los tipos I son endodérmicos, intralaríngeos y de extirpación endoscópica. Los tipo Ila son endodérmicos, extralaríngeos y los tipos IIB son endomesodérmicos y extralaríngeos. Los tipo II, para su extirpación completa, requieren un enfoque abierto ${ }^{21}$.

Entre los diagnósticos diferenciales del quiste vallecular aparecen el quiste del conducto tirogloso, el tiroides lingual, quiste dermoides, linfangioma, hemangioma y lipoma ${ }^{4,11}$.

La mayoría de las publicaciones recomiendan la realización de una nasofibroscopía como valoración diagnóstica inicial, por ser un examen fácil, rápido y poco invasivo. Tiene como limitación su disponibilidad y operador dependencia, especialmente para la zona de la vallécula y la base de lengua que en ocasiones es de dificultosa visuali- 
zación, requiriéndose una laringoscopía directa, la cual sigue siendo el gold standard ${ }^{11}$.

En nuestro caso, la rápida evolución de la insuficiencia respiratoria precipitó la instrumentalización de la vía aérea para soporte ventilatorio antes que la visualización fibroscópica. Posteriormente, la fibrobroncoscopía, descartó patología estructural bajo la glotis, sin embargo, debido a la presencia de la máscara laríngea, no fue posible la visualización del resto de la vía aérea supraglótica. Finalmente, fue la nasofibroscopía el examen que nos permitió hacer el diagnóstico preoperatorio.

Si es necesario caracterizar mejor la lesión, en caso de duda diagnóstica, la ecotomografía cervical resulta el método más útil para definir si el contenido es líquido o sólido. En términos generales la resonancia nuclear magnética es mejor definiendo las características de la lesión y sus límites, observándose una imagen hipointensa en T1, hiperintensa en T2 y que no se realza tras la administración de gadolinio; sin embargo, resulta poco práctica no sólo por el costo, sino principalmente porque requiere de sedación y el consiguiente manejo de vía aérea por un anestesista. La tomografía computarizada de cuello presenta similares desventajas, sumando además la irradiación ${ }^{12}$.

El tratamiento de los quistes valleculares, a diferencia de los quistes del sáculo laríngeo, reviste menos controversia. Leibowitz JM y cols en una serie de 11 casos reporta $27 \%$ de recurrencia, sin diferencias significativas entre marsupialización y excisión completa del quiste ${ }^{11}$. Suzuki $\mathrm{J}$ y cols en una revisión de 39 pacientes reportan 2,5\% de recurrencia con la marsupialización endoscópica ${ }^{4}$. En otra revisión de 33 casos, Hsieh LC y cols reporta $12 \%$ de recurrencia con marsupialización con láser $\mathrm{CO}_{2}$ y Tsai YT y cols, en 28 pacientes no reporta recurrencias con similar técnica ${ }^{22-23}$. Chen EY y cols y Prowse $S$ y cols, en series pequeñas de 7 y 6 casos, no reportan recurrencias empleando excisión completa y marsupialización endoscópica, respectivamente ${ }^{23-24}$. Gonik N y Smith LP reportan 3 casos de tratamiento con ablación por radiofrecuencia sin recurrencia ${ }^{25}$.

En términos generales, el procedimiento quirúrgico se realiza mediante visión directa con laringoscopio, teniendo presente no cubrir el quiste con la hoja de éste. Idealmente, la cirugía busca una extirpación completa de la cápsula del quiste. Si lo anterior no es posible, se debe realizar una marsupialización con técnica fría empleando microtijeras, electrocauterio o láser $\mathrm{CO}_{2}$. La aspiración con aguja fina no debe usarse como tratamiento definitivo dada su alta tasa de recidiva. En nuestro caso, se optó por una amplia marsupialización con técnica fría y hemostasia de los bordes con electrocauterización ${ }^{16,17}$.

\section{CONCLUSIÓN}

Los quistes valleculares son una rara causa de estridor congénito en el recién nacido. Es necesaria una alta sospecha clínica que permita realizar un diagnóstico presuntivo, el cual será confirmado mediante fibroscopía. La resolución quirúrgica debe ser meticulosamente programada idealmente junto con el anestesista, dado la dificultad intrínseca del manejo de la vía aérea. El abordaje terapéutico debe ser oportuno, tratando de realizar idealmente una exéresis completa 0 una marsupialización amplia del quiste.

\section{BIBLIOGRAFÍA}

1. L Zawadzka-Glos, M Frackiewicz, M Brzewski, A Biejat, M Chmielik, Difficulties in diagnosis of laryngeal cysts in children. Int $J$ Pediatr Otorhinolaryngol 2009; 73: 1729-31.

2. V Forte, J Warshawski, P Thorner, S Conley. Unusual laryngeal cysts in the newborn. Int $J$ Pediatr Otorhinolaryngol 1996; 37: 26-267.

3. Hsien WS, Yang PH, Wong KS, et al. Vallecular cyst: an uncommon cause of stridor in newborn infants. Eur J Pediatr 2000; 159(1-2): 79-81.

4. SuzukI J, Hashimoto S, Watanabe K, Takahashi K. Congenital vallecular cyst in an infant: case report and review of 52 recent cases. J Laryngol Otol 2011; 125: 1199-203.

5. LW DeSanto, KD Devine, LH Weiland. Cysts of the larynx classification. Laryngoscope 1970; 80: 145-76.

6. S PRowse. L. Knight / International Journal of Pediatric Otorhinolaryngology 2012; 76: 708-11.

7. Senthil R, Maskell S, Hartley B, Aladangady N. Blue episodes in a neonate. The Lancet 2009; 373: 1734. 
8. Khashu M, Osiovich H, Kozak F, Pelligra G, et al. Congenital epiglottic cyst presenting with severe airway obstruction at birth. J Perinato/2006; 26: 71-2.

9. MeSSNER AH. Congenital disorders of the larynx. In: Cummings CW, editor. Otolaryngology: head \& neck surgery. 4th edition. St. Louis (M0): Mosby, Inc.; 2005. p. 4223-40.

10. Bruce IA, Rothera MP. Upper airway obstruction in children. Paediatr Anaesth 2009; 19: 8899.

11. Leibowitz JM, Smith LP, Cohen MA, Dunham $B P$, ET AL. Diagnosis and treatment of pediatric vallecular cysts and pseudocysts. Int J Pediatr Otorhinolaryngol 2011; 75: 899-904.

12. Aziz aA, Abdullah Af, Ahmad RA. Two cases of congenital vallecular cyst: a reminder of the potentially fatal cause of upper airway obstruction in infants. Malays J Med Sci 2010; 17: 68-73.

13. Albert D, Boardman S, Soma M. Evaluation and management of the stridulous child. En: Flint $\mathrm{PH}$, Haughey BH, Lund VJ, Niparko JK, eds. Cummings Otolaryngology-Head and Neck Surgery. Maryland Heights, United States: Elsevier, 2010; 205: 2896-911.

14. Cheng KS, NG J, LI H, Hartigan PM. Vallecular cyst and laryngomalacia in infants: report of six cases and airway management. Anesth Analg 2002; 95: 1248-50.

15. Yao TC, Chiu CY, Wu KC, Wu LJ, et AL. Failure to thrive caused by the coexistence of vallecular cyst, laryngomalacia and gastroesophageal reflux in an infant. Int $J$ Pediatr Otorhinolaryngol 2004; 68: 1459-64.
16. DB Mitchell, BC Irwin, CM Bailey, JNG Evans. Cysts of the infant larynx. J Laryngol Otol 1987; 101: 833-7.

17. MW PaK, JKS Woo, CA van Hasselt. Congenital laryngeal cysts: current approach to management. J Laryngol Otol 1996; 110: 854-6.

18. BH Newman, JB Taxy, HI Laker. Laryngeal cysts in adults. A clinicopathologic study of 20 cases. Am J Clin Pathol 1984; 81: 715-20.

19. K Ramesar, C Albizzatı. Laryngeal cysts: clinical relevance of modified working classification. $J$ Laryngol Otol 1988; 102: 923-5.

20. C Arens, H Glanz, 0 Kleinsasser. Clinical and morphological aspects of laryngeal cysts. Eur Arch Otorhinolaryngol 1997; 254: 430-6.

21. V Forte, G Fuoco, A James. A new classification system for congenital laryngeal cysts. Laryngoscope 2004; 114: 1123-7.

22. Hsieh LC, Yang CC, Su CH, Lee KS, Chen BN, Wang LT. The outcomes of infantile vallecular cyst post C02 laser treatment. International Journal of Pediatric Otorhinolaryngology 2013; 77: 655-7.

23. Tsao YT, Lee LA, Fang TJ, Li HY. Treatment of vallecular cysts in infants with and without coexisting laryngomalacia using endoscopic laser marsupialization: fifteen year experience at a single-center. Int J Pediatr Otorhinolaryngol 2013; 77(3): 424-8.

24. Chen EY, LIM J, Boss EF, INGLIS AFJR, OU H, SIE KC, Manning SC, PeRins JA. Transoral approach for direct and complete excision of vallecular cysts in children. Int J Pediatr Otorhinolaryngol 2011; 75(9): 1147-51.

25. GoNIK N, SMITH LP. Radiofrequency ablation of pediatric vallecular cysts. Int J Pediatr Otorhinolaryngol 2012; 76(12): 1819-22. 